DRAFT VERSION JANUARY 24, 2020

Preprint typeset using $\mathrm{LAT}_{\mathrm{E}} \mathrm{X}$ style emulateapj v. 12/16/11

\title{
MODEL-INDEPENDENT CONSTRAINTS ON LORENTZ INVARIANCE VIOLATION: IMPLICATION FROM UPDATED GAMMA-RAY BURST OBSERVATIONS
}

\author{
Yu Pan ${ }^{1}$, Jingzhao Qi ${ }^{2}$, Shuo $\mathrm{CaO}^{3 *}$, Tonghua Lid ${ }^{3}$, Yuting Lid $^{3}$, Shuaibo Geng ${ }^{3}$, Yujie Lian ${ }^{3}$, And Zong-Hong \\ $\mathrm{ZHU}^{3}$ \\ Draft version January 24, 2020
}

\begin{abstract}
Astrophysical observations provide a unique opportunity to test possible signatures of Lorentz Invariance Violation (LIV), due to the high energies and long distances involved. In quantum theory of gravity, one may expect the modification of the dispersion relation between energy and momentum for photons, which can be probed with the time-lag (the arrival time delay between light curves in different energy bands) of Gamma-ray bursts (GRBs). In this paper, by using the detailed time-delay measurements of GRB 160625B at different energy bands, as well as 23 time-delay GRBs covering the redshifts range of $z=0.168-2.5$ (which were measured at different energy channels from the light curves), we propose an improved model-independent method (based on the newly-compiled sample of $H(z)$ measurements) to probe the energy-dependent velocity due to the modified dispersion relation for photons. In the framework of a more complex and reasonable theoretical expression to describe the time delays, our results imply that the intrinsic time lags can be better described with more GRBs time delay data. More importantly, through direct fitting of the time-delay measurements of a sample of GRBs, our limit on the LIV energy scale is comparable to that with unknown constant for the intrinsic time lag, much lower than the Planck energy scale in both linear LIV and quadratic LIV cases.
\end{abstract}

Keywords: astroparticle physics — gravitation — gamma-ray burst: general

\section{INTRODUCTION}

As one of the significant pillars of special/general relativity and particle physics, Lorentz invariance, which plays a very important role in modern physics, has been confirmed in all observations devoted to its testing to date (especially in solar system and colliders). However, during the last two decades a great attention has been paid to many quantum gravity (QG) theories with possible Lorentz invariance violation (LIV), in which the LIV will happen above the Planck energy scale $\left(E_{Q G} \approx\right.$ $E_{P l}=\sqrt{\hbar c^{5} / G} \simeq 1.22 \times 10^{19} \mathrm{GeV}$ due to the quantization of space-time (Mattingly 2005; Amelino-Camelia 2013)). Any possible violation of Lorentz invariance would have far-reaching consequences for our understanding of the Nature, i.e., the pillars of modern physics will be shocked and new physics is needed (Cao et al. 2018a). Therefore, the pursuit of testing the possible Lorentz invariance violation (LIV) at at much higher precision has continued in recent decades, concerning various astrophysical and cosmological observations.

Formulating and quantitatively interpreting the test of LIV is another question: an interesting proposal, in this respect, has been formulated in the frameworks of many quantum gravity (QG): at small spatial scales, a foamy structure of space-time predicted by QG theory will interact only with the high energy photons (Amelino-Camelia et al. 1998; Amelino-Camelia \& Piran 2001). In this case, the speed

\footnotetext{
${ }^{1}$ College of Science, Chongqing University of Posts and Telecommunications, Chongqing 400065, China;

2 Department of Physics, College of Sciences, Northeastern University, Shenyang 110004, China;

${ }^{3}$ Department of Astronomy, Beijing Normal University, 100875, Beijing, China; caoshuo@bnu.edu.cn
}

of light is varying at different energy range in vacuum, i.e., the high and low energy photons will not reach us at the same time. More specifically, the deformed velocity of light usually takes the form $v=c\left(1-s_{ \pm} E / E_{Q G}\right)$ where $E_{Q G}$ is effective QG energy scale, $s_{ \pm}$is a dimensionless parameter depending on the the particular QG model, and $c$ is the limiting speed of light on low energy scales, respectively. On low energy scales, $E \ll E_{Q G}$, the effect of LIV will be more obvious and the high energy photons propagate slower than low energy photons. Although the QG effect is expected to be very weak (since $E_{Q G}$ is typically close to the Planck energy scale), some effects of LIV are expected to increase with energy and over very long distances due to cumulative processes in photon propagation. Therefore, astrophysical searches provide sensitive probes of LIV and its potential signatures, such as the energy-dependent time delay and many other phenomena. Gamma-ray bursts (GRBs), the most luminous astrophysical events observable proposed as distance indicators at high redshift (Pan et al. 2013; Cao \& Zhu 2014), creates such opportunity to test the Lorentz invariance violation (LIV), through the precise measurement of unprecedented very-high-energy photons. Compared with commonly-used supernova Ia, the advantages of GRB lies in its high energy photons in the gammaray band (from $\mathrm{KeV}$ to $\mathrm{GeV}$ ), short spectral lags, and the propagation distance at cosmological scales. Such a natural laboratory provides a possibility of testing LIV, through the well-measured time delays between light curves in different energy bands caused by the LIV.

Recently, some advances have been made concerning the limits on LIV using different samples of GRBs (Ellis et al. 2003, 2006; Rodriguez Martínez \& Piran 2006; Jacob \& Piran 2007; Wei et al. 2018; Pan et al. 2015; Wei et al. 2017b; Zhang \& Ma 2015; Zhang et al. 
2018). The original idea of such studies of possible LIV constraints can be traced back to the papers of Ellis et al. (2003), which developed a method to analyze samples of GRBs with different redshifts and energy bands, by extracting time-dependent features from the GRB signals. It is worth noting that the intrinsic time delay and the linear term denotes LIV effect should be taken into account when one calculates the observed time delay $\Delta t$ (see Section 2 for details). Then, Ellis et al. (2008); Jacob \& Piran (2008) studied the possibility to test energy-dependent time delays through GRB measurements, which would result in strong sensitivity limits to LIV in the photon sector. Although no strong evidence of the LIV is currently supported by this astrophysical probe, one important issue should be reminded: the calculated time delay is strongly dependent on the cosmic expansion history (characterized by the Hubble parameter $H(z))$ and thus the pre-assumed cosmological model (the standard $\Lambda$ CDM model, etc) in all of the relevant works. For instance, it was found in Ellis et al. (2006) that there is no strong evidence of LIV and the effective QG energy scale can be determined at $E_{Q G} \geq 1.4 \times 10^{16} \mathrm{GeV}$, in the framework of the concordance $\Lambda \mathrm{CDM}$ model with all of the model parameters taken from the typical results from WMAP observations. A weak evidence of LIV was also noticed and discussed in Biesiada \& Piórkowska (2009), which studied the LIV by applying the quintessence and Chaplygin Gas model to the observational GRB time delays. However, it should be noted that in the above analysis all the values of the corresponding model parameters have been fixed and the degeneracies among cosmological parameters were neglected. Further progress in this direction has recently been achieved by Pan et al. (2015); Wei et al. (2018) in two recent paper, which respectively constrain different cosmological (or cosmographical) parameters together with the LIV parameters by using the observational data. While comparing the results from their works, no apparent evidence of LIV and weak hints for LIV are respectively reported. Up to now, there existed several explanations of this weak hints of possible LIV. First of all, it may be just a statistical result produced by the limited amount of observational data available. In order to draw firm and robust conclusions, one will need to minimize statistical uncertainties by increasing the depth and quality of observational data sets. Secondly, to our best knowledge, one or more particular cosmological models have been assumed in (almost) all of the relevant works in the literature, which makes the results on LIV in those works model-dependent and hence not so robust in fact. Therefore, reconstruction of the cosmic expansion history may strongly influence the estimated values of the LIV parameters. Thirdly, one cannot ignore the fact that the weak hints of possible LIV may be brought by some caveats in the LIV parametrization.

In this context, it is clear that collection of more complete observational data concerning time-delay measurements does play a crucial role. The purpose of our paper is to show how the combination of the most recent and significantly improved time-delay measurements of GRB 160625B at different energy bands, as well as 34 time-delay GRBs covering the redshifts range of $z=0.168-4.3$ (which were measured at different energy channels from the light curves) can be used to probe possible signal of LIV and set limits on the value of $E_{Q G}$ (Ellis et al. 2006). More importantly, compared with the previous works using the luminosity distances from type Ia supernova, we will use instead, cosmological distances covering the GRB redshift range derived in a cosmological-model-independent way from Hubble parameter measurements using Gaussian processes (GP), based on the newly-compiled sample of $H(z)$ measurements (Wei et al. 2017a). In order to discuss the LIV in a general framework, a more complex and reasonable theoretical expression will be considered in our analysis. We expect that the newest measurements of GRBs combined with non-parametric distance reconstruction from the most recent $H(z)$ data will shed much more light on the possible hint for LIV at higher redshifts. This paper is organized as follows. In Section 2, we briefly describe the methodology of the intrinsic time lag and the time delay induced by LIV. Then, in Section 3 we introduce the time delay data from GRBs, and the observational data of the Hubble parameters used in our analysis. The results and corresponding discussion are presented in Section 4. Finally, we summarize our conclusions in Section 5.

\section{THE LORENTZ INVARIANCE VIOLATION}

As one of the successful predictions of general relativity in the past decades, the energy dependent velocity of light is $E^{2}=p^{2} c^{2}$, by assuming constant speed of light and the validity of Lorentz invariance. However, on low energy scales the introduction of a Lorentz violating term in the standard model can induce modifications to the particle dispersion relation

$$
E^{2}=p^{2} c^{2}\left[1-s_{ \pm}\left(\frac{E}{E_{Q G}}\right)^{n}\right]
$$

where $s_{ \pm}= \pm 1\left(s_{ \pm}=+1\right.$ or $s_{ \pm}=-1$ stands for a decrease or an increase in photon velocity with an increasing photon energy), while the $n$ parameter represents the leading order (linear term or quadratic term) of the correction from the underlying theory $(n=1$ corresponds to the Double Special Relativity (Magueijo \& Smolin 2002; Amelino-Camelia 2002; Amelino-Camelia. 2010) and $n=2$ corresponds to Extra-Dimensional Theories (Sefiedgar et al 2011) or Harava-Lifshitz Gravity (Horava 2009a,b; Vacaru 2010; Blas \& Sanctuar 2011)). Now the energy-dependent speed of photon can be written as

$$
v=\frac{\partial E}{\partial p}=c\left[1-s_{ \pm} \frac{n+1}{2}\left(\frac{E}{E_{Q G}}\right)^{n}\right]
$$

Note that we only consider the case of $s_{ \pm}=+1$ in the present work, since the high-energy photons travel slower than their low-energy counterparts. On the other hand, the linear term $(n=1)$ obviously dominate the dispersion relation for $E \ll E_{Q G}$. In order to have a better extension and discussion, in this analysis we also consider the second case with quadratic term $(n=2)$, which is different from most of the relevant works in the literature.

Now over long distances due to cumulative processes in photon propagation, one can get the LIV-induced time delay between photons with high energy and low energy (Jacob \& Piran 2007, 2008; Biesiada \& Piórkowska 2007, 
2009)

$$
\Delta t_{\mathrm{LIV}}=\frac{1+n}{2 H_{0}} \frac{E^{n}-E_{0}^{n}}{E_{Q G, n}^{n}} \int_{0}^{z} \frac{\left(1+z^{\prime}\right)^{n} d z^{\prime}}{h\left(z^{\prime}\right)}
$$

where $H_{0}$ is the present value of the Hubble function, $h(z)=H(z) / H_{0}$ is a dimensionless expansion rate dependent on redshift $z$. From observational point of view, for a cosmic transient source (e.g. GRB), the observed time delay between two different energy bands should include five terms

$$
\Delta t=\Delta t_{\mathrm{LIV}}+\Delta t_{\mathrm{int}}+\Delta t_{\mathrm{spe}}+\Delta t_{\mathrm{DM}}+\Delta t_{\mathrm{gra}}
$$

where $\Delta t_{\mathrm{spe}}$ is related to the potential time delay due to special relativistic effects if photons have a non-zero rest mass (Gao et al. 2015), while $\Delta t_{\mathrm{DM}}$ and $\Delta t_{\text {gra }}$ respectively denote the time delay contribution from the dispersion by the line-of-sight free electron content and the gravitational potential along the propagation path of photons if the Einsteins equivalence principle (EEP) is violated (Wei et al. 2015). However, the effect of the three terms is negligible for GRB photons, following the recent analysis of Wei et al. (2015); Gao et al. (2015). The first term, $\Delta t_{\text {LIV }}$ represents the LIV-induced time delay, while the second term $\Delta t_{\text {int }}$ quantifies the intrinsic time delay describing that photons with high and low energies do not leave the source simultaneously. In order to account for the unknown intrinsic time lags, the unknown intrinsic time delays of GRBs are usually specified by including a parameter $b$ in the rest-frame of the source, which can be written as $\Delta t_{\mathrm{int}}=b(1+z)$ when the cosmic expansion is taken into account (Ellis et al. 2006; Pan et al. 2015). More recently, it was proposed in the recent analysis that the assumption that the intrinsic time lag between the lowest energy band and any other high energy bands increases with the energy $E$ is more reasonable (Wei et al. 2017b). In this paper we will adopt this more reasonable formulation of the intrinsic energy-dependent time lag, in the form of an approximate power-law function:

$$
\Delta t_{\mathrm{int}}(E)=\tau\left[\left(\frac{E}{\mathrm{keV}}\right)^{\alpha}-\left(\frac{E_{0}}{\mathrm{keV}}\right)^{\alpha}\right],
$$

where $\tau$ and $\alpha$ are two positive free parameters, and $E_{0}$ is the low energy band. Therefore, the theoretical time delay between different energy bands of GRBs can be expressed as

$$
\begin{aligned}
\Delta t_{\mathrm{th}}= & \Delta t_{\mathrm{LIV}}+\Delta t_{\mathrm{int}}(1+z) \\
= & \frac{1+n}{2 H_{0}} \frac{E^{n}-E_{0}^{n}}{E_{Q G, n}^{n}} \int_{0}^{z} \frac{\left(1+z^{\prime}\right)^{n} d z^{\prime}}{h\left(z^{\prime}\right)} \\
& +\tau\left[\left(\frac{E}{\mathrm{keV}}\right)^{\alpha}-\left(\frac{E_{0}}{\mathrm{keV}}\right)^{\alpha}\right](1+z) .
\end{aligned}
$$

Note that there are three parameters in this model $\left\{E_{Q G}\right.$, $\tau, \alpha\}$. For convenience, we define a parameter $K(z)$ as (Pan et al. 2015)

$$
K(z)=\int_{0}^{z} \frac{\left(1+z^{\prime}\right)^{n} d z^{\prime}}{h\left(z^{\prime}\right)},
$$

which is related to the measurements of cosmic distances. As is mentioned above, almost all of the previous test

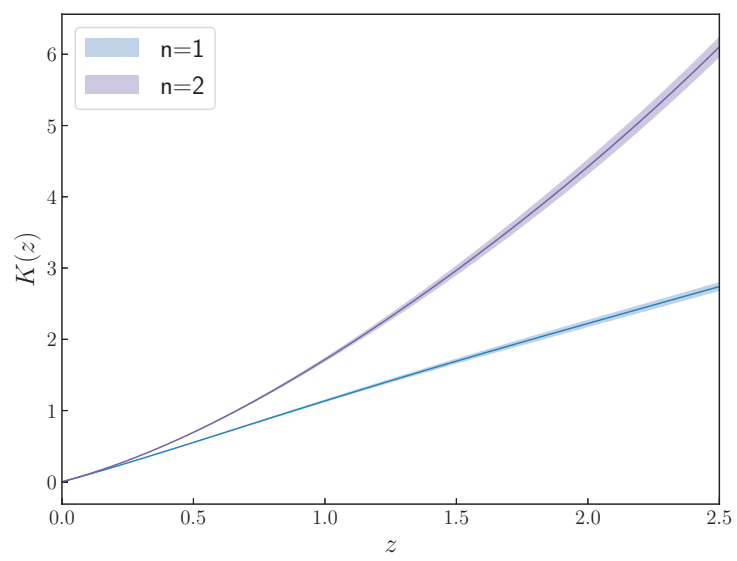

Figure 1. The distribution of reconstructed $K(z)$ with redshifts, in the two cases of $n=1$ and $n=2$.

Table 1

The $1 \sigma$ constraints on $E_{Q G}, \tau$ and $\alpha$ for different LIV models $(n=1, n=2)$.

\begin{tabular}{|c|c|c|}
\hline Model parameter & $n=1$ & $n=2$ \\
\hline $\log E_{Q G}(\mathrm{GeV})$ & $14.523_{-0.025}^{+0.022}$ & $8.79_{-0.0097}^{+0.0097}$ \\
\hline$\tau$ & $0.00067_{-0.00005}^{+0.00005}$ & $0.0000018_{-0.00000009}^{+0.00000009}$ \\
\hline$\alpha$ & $1.0556_{-0.0046}^{+0.0046}$ & $2.0209_{-0.0034}^{+0.0034}$ \\
\hline
\end{tabular}

of LIV used the cosmological models to calculate the distance-like parameter $K(z)$, based on the General Relativity frame without LIV. Therefore, a more reasonable approach will be applied to construct $K(z)$, which is model-independent, rather than assuming a particular cosmo-logical model as in the literature.

\section{OBSERVATIONAL DATA}

In this paper, we use the time delay data of GRB $160625 \mathrm{~B}$ at different energy bands (whose redshift is $z=1.41$ ) (Xu et al. 2016) and 34 time-delay GRBs (with the redshifts spanning from $z=0.168$ to $z=4.3$ ), which were derived from time lags between different energy channels measured from the light curves.

The GRB 160625B was triggered and located twice by the Fermi Gamma-Ray Burst Monitor (GBM) (Burns 2016), with a sharp increase in the rate of high-energy photons and an onboard trigger on a bright pulse detected by the fermi Large Area Telescope (LAT). The gamma-ray light curve of GRB 160625B consists of three dramatically different isolated sub-bursts, while the spectral time lags are obtained from the light curves in the 15 - $350 \mathrm{keV}$ energy band with respect to a total duration of $\sim T_{90}=770 \mathrm{~s}$ (Zhang et al. 2016b). One should note that the second sub-burst of GRB 160625B is very bright, which made it possible to easily extract its light curves in different energy bands. The observed time lags measured from the energy-dependent light curves are listed in Table 1 of Wei et al. (2017b). Meanwhile, based on the techniques from signal processing such as wavelet analysis to identify and correlate genuine features in the intensities observed in different energy bands (Ellis et al. 2003), a time delay data set from 35 GRBs was compiled by Ellis et al. (2006), with known redshifts from 
$z=0.168$ to $z=6.29$. All of the data were shown in Table 1 of Ellis et al. (2006) including the 9 time-delay of GRBs from light curves whose time resolution is $64 \mathrm{~ms}$ and redshifts span from $z=0.835$ to $z=3.9$ observed by BATSE spectral channels, the HETE data with 15 light curves whose time resolution is $164 \mathrm{~ms}$ and redshifts span from $z=0.168$ to $z=3.372$, and the SWIFT data with 10 light curves whose time resolution is $64 \mathrm{~ms}$ and redshifts span from $z=0.258$ to $z=4.3$. The spectral time lags are obtained from the light curves in the $25-320$ $\mathrm{keV}$ energy band. The observed time lags measured from the energy-dependent light curves are listed in Table 1 of Ellis et al. (2006).

On the other hand, we also use Gaussian processes to reconstruct the function $K(z)$ from observational $H(z)$ data directly. Such idea was first discussed in Holsclaw et al. (2010) and then extensively applied in more recent papers to test the cosmological parameters (Cao et al. 2017a, 2018b), spatial curvature of the Universe (Cao et al. 2019; Qi et al. 2019a), and the speed of light at higher redshifts (Cao et al. 2017b). In this process, the reconstructed function $f(x)$ at different points $x$ and $\tilde{x}$ are correlated by a covariance function $k(x, \tilde{x})$ (Seikel et al. 2012). The commonly used function is the squared exponential covariance, whose advantage is infinitely differentiable, which only depends on two hyper parameters $\ell$ and $\sigma_{f}$. Both $\ell$ and $\sigma_{f}$ would be trained to determine the specific value by GP code self with the observational data. Therefore, the GP method does not specify any form of $f(x)$ and is model-independent. Moreover, we use the publicly available code called the GaPP (Gaussian Processes in Python) 4 reconstruct the profile of $H(z)$ function up to the redshifts $z=2.5$, which has been widely used in various studies (Yang et al. 2015; Qi et al. 2016; Zhang \& Xia 2016). In this paper, we use the newest Hubble parameter $(H(z))$ data set, which consist of 31 measurements from the differential ages of passively evolving galaxies and 10 measurements via the detection of radial BAO features (Zheng et al. 2019). One should note that the limited redshift range of $H(z)$, i.e., $z \sim 2.5$ implies that only a limited number of known GRBs can be used. This selection leaves us with the detailed time-delay measurements of GRB 160625B (Wei et al. 2017b) and 23 time-delay GRBs summarized in Ellis et al. (2006).

\section{RESULTS AND DISCUSSION}

Using the aforementioned GP, one is able to reconstruct the profile of $K(z)$ function up to the redshift of $z=2.5$ (assuming flat universe). The results are shown in Fig. 11 in the framework of two cases with $n=1$ and $n=2$. Based on the reconstructed functions of $K(z)$, we determine the parameters $\left(E_{Q G}, \tau\right.$ and $\left.\alpha\right)$ characterizing LIV by minimizing the $\chi^{2}$ objective function

$$
\chi_{G R B}^{2}=\sum_{i=1}^{N_{G R B}}\left[\frac{\Delta t_{t h}\left(E_{Q G}, \tau, \alpha\right)-\Delta t_{o b s}}{\sigma_{\Delta t}}\right]^{2},
$$

where $\Delta t_{t h}$ denotes the theoretical time delays of GRB, and $\Delta t_{o b s}$ is the observational counterpart with the corresponding $1 \sigma$ uncertainty $\left(\sigma_{\Delta t}\right)$. We apply the Monte

\footnotetext{
${ }^{4}$ http://www.acgc.uct.ac.za/ seikel/GAPP/index.html
}

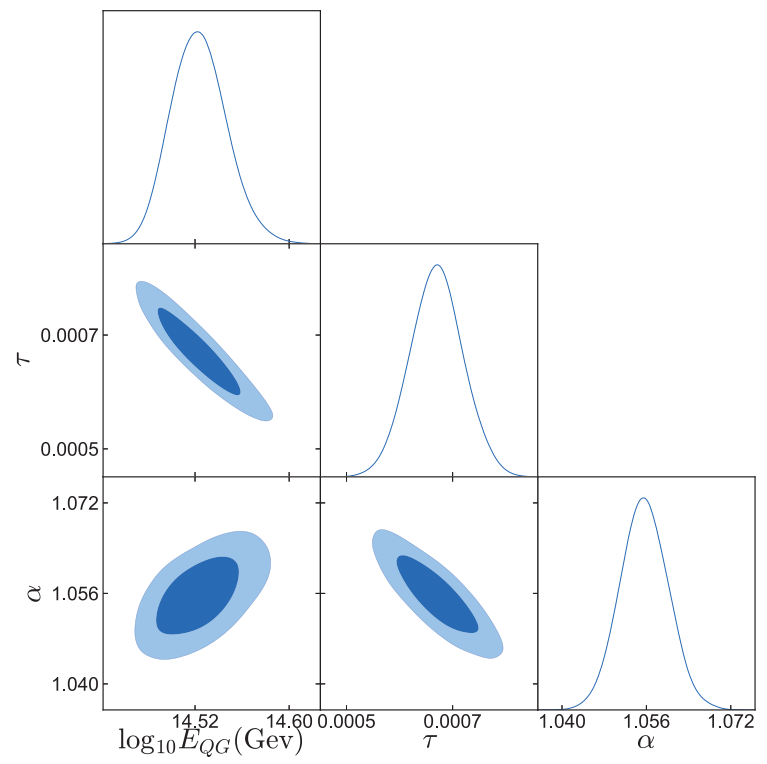

Figure 2. The $1 \mathrm{D}$ probability distribution of each parameter and the $2 \mathrm{D}$ confidence contours for the parameters $E_{Q G}, \tau$ and $\alpha$ (the linear LIV case, i.e., $n=1$ ).

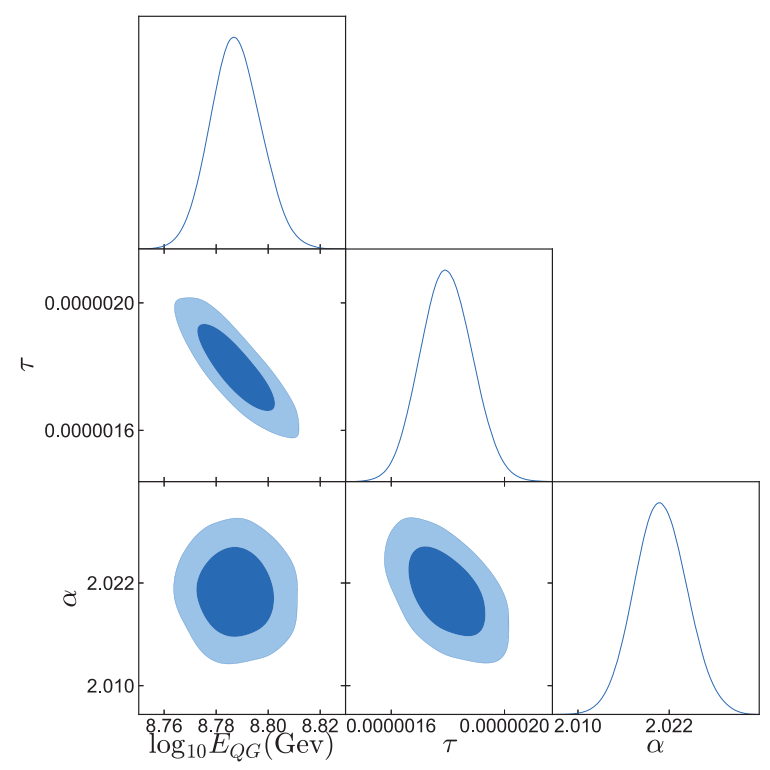

Figure 3. The 1D probability distribution of each parameter and the $2 \mathrm{D}$ confidence contours for the parameters of $E_{Q G}, \tau$ and $\alpha$ (the quadratic LIV case, i.e., $n=2$ ).

Carlo Markov Chain (MCMC) method (Lewis \& Bridle 2002) with 8 chains and obtain the marginalized $1 \sigma$ and $2 \sigma$ constraints. Performing fits to different scenarios ( $n=1$ and $n=2$ ) on the GRB sample, we obtain the results displayed in Table 1 . The marginalized probability distribution of each parameter and the marginalized 2-D confidence contours are presented in Figs. 1-2.

Let's start from the first case by fitting the observed time-delay data with the linear LIV case (i.e., $n=1$ ). Performing fits on the the time-lag (the arrival time delay between light curves in different energy bands) of GRBs, we obtain the following best-fit values and corresponding 
$1 \sigma$ uncertainties (68.3\% confidence level):

$$
\begin{aligned}
& \log \mathrm{E}_{\mathrm{QG}, 1}(\mathrm{GeV})=14.523_{-0.025}^{+0.022}, \\
& \tau=0.00067_{-0.00005}^{+0.00005} \\
& \alpha=1.0556_{-0.0046}^{+0.0046}
\end{aligned}
$$

The resulting constraints on $E_{Q G, 1}, \tau$ and $\alpha$ are shown in Fig. 2. Thanks to the improved statistical technique and the use of a more complete data set, the result of our present analysis is significantly stronger and more robust than that in Ellis et al. (2006). Since Lorentz invariance plays an important role in modern physics, it is interesting to to distinguish the possible time delay induced by the LIV effect from any source-intrinsic time lag in the emission of photons at different energies. On the one hand, tighter estimates of the intrinsic energy-dependent time lag is obtained. We find that the best-fitted value of the parameter $\tau$ is a small number, $|\tau| \sim 6 \times 10^{-4}$. On the other hand, different from the previous procedure assuming an unknown constant to characterize the intrinsic time lag for all GRBs, our results show that the intrinsic lag has a positive dependence on the photon energy. More specifically, compared with the previous results obtained on the individual source GRB 160625B (Wei et al. 2017b), our full GRB sample analysis has yielded improved constraints on this meaningful physical parameter, $\alpha \sim 1.05$. It should be noted that in the framework of LIV, such positive correlation between the lag and the energy will gradually become an anticorrelation at the high energy scales, since high energy photons travel slower than low energy photons in vacuum (Wei et al. 2017b). More importantly, more rigorous quantitative analysis supports the limit of the QG energy scale on the linear LIV case, $E_{Q G, 1} \geq 0.3 \times 10^{15} \mathrm{GeV}$, four orders of magnitude below the Planck energy scale. Such analysis is comparable to the limit found from Ellis et al. (2006) with unknown constant for the intrinsic time lag for the linear LIV case, through direct fitting of the time-delay measurements of a sample of GRBs.

Next, we consider the quadratic LIV case (i.e., $n=2$ ) to fit the observed time-delay data data. Focusing on the second case with the quadratic term, the best fit is

$$
\begin{aligned}
& \log \mathrm{E}_{\mathrm{QG}, 2}(\mathrm{GeV})=8.79_{-0.0097}^{+0.0097}, \\
& \tau=0.0000018_{-0.00000009}^{+0.0000009} \\
& \alpha=2.0209_{-0.0034}^{+0.0034} .
\end{aligned}
$$

Marginalized probability distributions for each parameter and marginalized 2D $68 \%$ confidence contours are presented in Fig. 2. The estimation of these LIV parameters is briefly summarized in Table 1. Comparing constraints based on a different value for the $n$ term, we see the two parameters quantifying the intrinsic time lag in different energy bands are in general agreement with the corresponding quantities for the linear LIV case. More specifically, the best-fit value of $\tau$ still tends to be zero $\left(|\tau| \sim 6 \times 10^{-6}\right)$, while its positive dependency on the photon energy $(\alpha \sim 2.02)$ is consistent with the observational data within $1 \sigma$ confidence region. In broad terms, this reveals that both cases are adequate to represent the time lag of GRBs. With the best-fit values of $\log \mathrm{E}_{\mathrm{QG}, 2}(\mathrm{GeV})$ as well as its $1 \sigma$ error bar, the $1 \sigma$ confidence-level lower limit on LIV is
$E_{Q G, 2} \geq 0.6 \times 10^{9} \mathrm{GeV}$ and the sensitivity of this analysis might have been expected to be two orders of magnitude greater than in Wei et al. (2017b). In this way, our results can exclude the energy scale of the new LIV physics, $E_{Q G, 2}$, to greater than $10^{9} \mathrm{GeV}$. The most conservative limit on the violation of Lorentz invariance that we find is two orders of magnitude below the current best limit from the single GeV photon of GRB 090510 Abdo et al. 2009b; Vasileiou et al. 2015). Note that, although some stronger upper limits on a modification of the photon dispersion relation have been reported in the literature, any analysis of a single source can only be regarded as indicative, due to unknown systematic uncertainties associated with unknown intrinsic spectral properties of any given GRB (Ellis et al. 2006). In order to establish a rigorous limit, one must focus on the differentiation between intrinsic and propagation effects in the time-delay measurements, which can be done robustly only by analyzing a sizeable statistical sample of GRBs. Now it is worthwhile to make some general comments on the reliability of the resulting constraints on LIV. On the one hand, the analysis of the intrinsic time lag performed here is important for studying the flight time differences from the astronomical sources to test the LIV effect. Different from the previous model with an unknown constant for the intrinsic time lag (Ellis et al. 2006), we use a more complex and reasonable theoretical expression to describe the intrinsic energy-dependent time lags (Wei et al. 2017b). Meanwhile, compared with the previous works using the luminosity distances from type Ia supernova to quantify LIV effect (Pan et al. 2015), we use instead, cosmological distances derived in a cosmological model-independent way from $H(z)$ measurements using Gaussian processes (GP) (Wei et al. 2017a; Zheng et al. 2019). By considering the contribution of both the intrinsic time lag and the lag by the LIV effect, our analysis indicates that the intrinsic time lags can be obviously better described with more GRBs time delay data. More importantly, it is possible to give robust limits on LIV through direct fitting of the spectral lag data of a GRB.

There are several sources of systematics that we do not consider in this paper and remain to be addressed in future analysis. First of all, we have learned from our analysis that the reliability of the resulting constraints on LIV strongly depends on a good knowledge of the intrinsic time lag, i.e., photons with high and low energies do not leave the GRB simultaneously. Although the problems associated with the intrinsic time delay can be handled better with the new formulation of $\Delta t_{i n t}$, such astrophysical term is still difficult to predict since it depends only on good understanding of the physics of source evolution (Ellis et al. 2008). More importantly, the strong correlation between the parameters describing intrinsic and propagation time-lags have revealed new systematic issues, which can be clearly seen from Fig. 2-3. Therefore, in our approach the available GRB data can be used conservatively to set a lower limit on any Lorentz Invariance Violation (LIV). Further progress in this direction has recently been achieved by Ellis et al. (2006), which suggested that a supposedly more reliable subs-ample of GRBs with a spread of different measured redshifts are advantageous, from the point of view of demonstrating the absence of any destructive interference between intrinsic and propagation effects. In this aspect, the re- 
duction of the above potential systematic bias should turn to better understanding of the internal dynamics of GRBs or available data extending to much higher energies (Ellis et al. 2008). Secondly, the other source of systematic uncertainty comes from the reconstruction of the function $K(z)$. Different from the previous studies assuming a particular cosmological model (e.g. $\Lambda$ CDM) (Wei et al. 2017b), we have applied one particular nonparametric method based on Gaussian processes to reconstruct the function $K(z)$ from observational $H(z)$ data. The only way to minimize this unknown systematic uncertainty is to search for more efficient distance reconstruction technique or model-independent methodology. This problem has also been recognized and discussed in many recent works (Zou et al. 2018), with a heuristic suggestion that with the so-called cosmography (one of the powerful model-independent approaches), one can analyze the evolution of the universe without assuming any underlying theoretical model. As a final remark, we point out that the time-lags discussed in this paper are derived from the sharp features observed in the intensities of radiation with different energies, identified by various wavelet techniques for different GRB subsamples. Therefore, the follow up engaging the observations of more GRBs with higher temporal resolutions and more high energy photons may make it less susceptible to such systematic errors.

\section{CONCLUSIONS}

Modern ideas in quantum gravity predict the possibility of Lorenz Invariance Violation (LIV), which reveals itself by energy dependent modification of standard relativistic dispersion relation. Following this direction, time of flight delays in photons emitted by astrophysical sources located at cosmological distances can become a valuable tool for setting limits on LIV theories. In this paper we discuss an improved model-independent method to constrain the energy-dependent velocity due to the modified dispersion relation, based on the detailed time-delay measurements of GRB 160625B at different energy bands and 23 time-delay GRBs covering the redshifts range of $z=0.168-2.5$. Two parametric expressions are considered to describe the LIV-induced time delay between photons with high energy and low energy (linear and quadratic LIV cases). Here we summarize our main conclusions in more detail:

- In most of the relevant works on LIV, the intrinsic time-lag of GRBs is actually oversimplified by assuming $\Delta t_{\text {int }}=b(1+z)$, which means that all GRBs have the same intrinsic time delay in the source frame. Meanwhile, one or more particular cosmological models have been assumed in the literature, which makes the results on LIV modeldependent. In this paper, we turn to a more complex and reasonable theoretical expression to describe the energy-dependent intrinsic time-lag, while the cosmic expansion history in the LIV time delay is reconstructed from observational $H(z)$ data based on model-independent Gaussian processes.

- Our results show that the intrinsic time lags can be better described with more GRBs time delay data. Instead of assuming an unknown constant for the intrinsic time lag, we argue that the intrinsic lag has a positive dependence on the photon energy for both linear LIV and quadratic LIV cases. More importantly, the strong correlation between the parameters describing intrinsic and propagation time-lags have been revealed in our analysis, which indicates that a more realistic assumption for the intrinsic time-lag of GRBs is important to robustly constrain the possible LIV. In the linear LIV and quadratic LIV cases, one can limit the LIV energy scale at the level of much lower than the Planck energy scale: $E_{Q G, 1} \geq 0.3 \times 10^{15} \mathrm{GeV}$ and $E_{Q G, 2} \geq 0.6 \times 10^{9} \mathrm{GeV}$. Although some stronger upper limits on LIV have been reported from the single GeV photon of a single source, our approach can be used conservatively to set a lower limit on any LIV.

- In conclusion, our analysis demonstrates that timelags in emissions from GRBs can already now be used to set limit on the Violation of Lorentz Invariance, in the framework of a more complex and reasonable formulation to describe the LIV effect. One may say that the approach initiated in Ellis et al. (2003, 2008); Jacob \& Piran (2008) can be further developed to analyze a larger sample of GRBs with different redshifts and energy bands. Fits on the phenomenological formula obtained in our analysis, if confirmed by future investigation of GRB timedelay observations, will offer additional constraints for possible LIV with extragalactic sources.

- Finally, it is reasonable to expect that more GRBs with higher temporal resolutions and more high energy photons (which induce large time delays) can be used to test the possible LIV. In order to establish a rigorous limit, we also pin hope on a significantly larger sample of LIV probes at much higher redshifts, including the photon time-delay measurements from objects like Active Galactic Nuclei(AGN) (Albert et al. 2008) and gravitational waves (Passos et al. 2016). With such complementary probes, combined with Hubble parameter measurements obtained covering the redshifts range of $0.1 \leq z \leq 5.0$ in the near future (Yu \& Wang 2016; Weinberg et al. 2013), we can further investigate constraints on the LIV effect and eventually probe the deep physics behind LIV, i.e., the quantum gravity theories (string theory, loop quantum gravity, and doubly special relativity) and field theory frameworks for LIV (the so-called Standard-Model Extension) (Kostelecky \& Mewes 2008; Kostelecky \& Russell 2011; Kislat \& Krawczynski 2015).

\section{ACKNOWLEDGMENTS}

This work was supported by National Key R\&D Program of China No. 2017YFA0402600, the National Natural Science Foundation of China under Grants Nos. 11690023, 11373014, and 11633001, the Strategic Priority Research Program of the Chinese Academy of Sciences, Grant No. XDB23000000, the Interdiscipline Research Funds of Beijing Normal University, and 
the Opening Project of Key Laboratory of Computational Astrophysics, National Astronomical Observatories, Chinese Academy of Sciences. J.-Z. Qi was supported by China Postdoctoral Science Foundation under Grant No. 2017M620661. M. Biesiada was supported by Foreign Talent Introducing Project and Special Fund Support of Foreign Knowledge Introducing Project in China. He is also grateful for support from Polish Ministry of Science and Higher Education through the grant DIR/WK/2018/12. Y. Pan was supported by the Scientific and Technological Research Program of Chongqing Municipal Education Commission (Grant no. KJ1500414); and Chongqing Municipal Science and Technology Commission Fund (cstc2015jcyjA00044, and cstc2018jcyjAX0192).

\section{REFERENCES}

Abdo, A. A., Ackermann, M., Ajello, M., et al. 2009, Nature, 462, 331

Abdo, A. A., Ackermann, M., Arimoto, M., et al. 2009, Science, 323,1688

Abdo, A. A., Ackermann, M., Arimoto, M., et al. 2014, A\&A, $571, \mathrm{~A} 16$

Albert, J., Ellis, J., Mavromatos, N. E., et al. 2008, PLB, 668, 253

Amanullah, R., Lidman, C., Rubin, D., et al. 2010, ApJ, 716, 712

Amelino-Camelia, G., World Scientific Publishing Co. Pte. Ltd., 2010. ISBN:9789814287333, 123-170

Amelino-Camelia, G., Ellis, J., Mavromatos, N. E., Nanopoulos, D. V., Subir S. 1998, Nature, 393, 763

Amelino-Camelia, G. 2001, PLB, 510, 255

Amelino-Camelia, G., Piran, T. 2001, PRD, 64, 036005

Amelino-Camelia, G. 2002, IJMPD, 11, 35

Amelino-Camelia, G., Laemmerzahl, G., Mercati, F., Guglielmo M. T. 2009, PRL, 103, 171302

Amelino-Camelia, G. 2013, Living Rev. Rel, 16, 5

Armendariz-Picon, C., Mukhanov, V., Steinhardt, P. J. 2001, PRD, 63, 103510

Astier, P., Guy, J., Regnault, N., et al. 2006, A\&A, 447, 31

Baukh, V., Zhuk, A., Kahniashvili, T. 2007, PRD, 76, 027502

Blas, D., Sanctuary, H. 2011, PRD, 84, 064004

Beutler, F., Blake, C., Colless, M., et al. 2011,MNRAS, 416, 3017

Biesiada, M., Piórkowska, A. 2007, JCAP, 0705, 011

Biesiada, M, Piórkowska, A. 2009, CQG, 26, 125007

Biller, S. D., Breslin, A. C., Carson, M., et al. 1999, PRL, 83, 2108

Chris, B., Tamara, D., Gregory, B. P., David, P., Sarah, B. 2011, MNRAS, 418, 1707

Borriello, E., Chakraborty, S., Mirizzi, A., Serpico, P. D. 2013, PRD, 87, 116009

Burns, E. 2016, GRB Coordinates Network, 19581

Caldwell, R. R., Dave, R.,Steinhardt, P. J. 1998, PRL, 80, 1582

Caldwell, R. R. 2002, PLB, 545, 23

Caldwell, R. R., Kamionkowski, M., Weinberg, N. N. 2003, PRL, 91, 071301

Cao, S., Zhu, Z. H. 2011, Science in China G: Physics and Astronomy, 54, 2260

Cao, S., Pan, Y., Biesiada, M., Godlowski, W.,Zhu, Z. H. 2012a, JCAP, 1203, 016

Cao, S., Covone, G., Zhu, Z.-H. 2012b, ApJ, 755, 31

Cao, S., Zhu, Z.-H. 2014, PRD, 90, 083006

Cao, S., Biesiada, M., Jackson, J., et al. 2017, JCAP, 02, 012

Cao, S., Zheng, X. G.,Biesiada, M., et al. 2017, A\&A, 606, A15

Cao, S., Qi, J. Z., Biesiada, M., et al. 2018a, ApJ, 867, 50

Cao, S., Zheng, X. G., Biesiada, M., Qi, J. Z., Chen, Y. 2018, EPJC, 78, 749

Cao, S., Qi, J. Z., Biesiada, M., et al. 2019, PDU, 24, 100274

Chevallier, M., Polarski, D. 2001, IJMPD, 10, 213

Chiba, T. 2002, PRD, 66, 063514

Eisenstein, D. J., Hu, W. 1998, ApJ, 496, 605

Eisenstein, D. J., Zehavi, I., Hogg. D. W, et al. 2005, ApJ,, 633, 560

Ellis, J., Mavromatos, N. E., Nanopoulos, D. V., Sakharov, A. S. 2003, A\&A, 402, 409
Ellis, J., Mavromatos, N. E., Nanopoulos,D.V., Sakharov, A. S., Sarkisyan, E. K. G. 2006, Astroparticle Physics, 25, 402

Ellis, J., Mavromatos, N. E., Nanopoulos, D. V., Sakharov, A. S. 2008, A\&A, 402, 409

Feng, B., Wang, X. L., Zhang, X. M. 2005a, PLB, 607, 35

Feng, B., Li, M. Z., Piao, Y. S., Zhang, X. M. 2006, PLB, 634, 101 Gao, Q., Gong, Y. 2014, CQG, 31, 105007

Gao, H., Wu, X. F., Mészá,P. 2015, ApJ, 810, 121

Gong, Y. G., Gao, Q., Zhu, Z.-H. 2013, MNRAS, 430, 3142

Gong, Y. G., Gao, Q. 2014, EPJC, 74, 2729

Guo, Z. K., Piao, Y. S., Zhang, X. M., Zhang, Y. Z. 2005, PLB, 608,177

Guo, Z. K., Huang, Q. G., Cai, R. G., Zhang, Y. Z. 2012, PRD, 86,065004

Guo, Z. K., Hu, J. W. 2013, PRD, 87, 123519

Hicken, M., Wood-Vasey, W. M., Blondin, S., et al. 2009, ApJ, 700,1097

Holsclaw, T., Alam, U., Sanso, B., et al. 2010, PRL, 105, 241302

Horava, P. 2009, JHEP, 03, 020

Horava, P. 2009, PRD, 79, 084008

Hu, W., Sugiyama, N. 1996, ApJ, 471, 542

Jacob, U., Piran, T. 2007, Nature Physics, 3, 87

Jacob, U., Piran, T. 2008, JCAP, 0801, 031

Kaaret, P. 1999, A\&A, 345, L32

Kislat, F., Krawczynski, H. 2015, PRD, 92, 045016

Komatsu, E., Dunkley, J., Nolta, M. R., et al. 2009, ApJS, 180, 330

Komatsu, E., Smith, K. M., Dunkley, J., et al. 2011, ApJS, 192, 18

Kostelecky, V. A., Mewes, M. 2008, ApJL, 689, L1

Kostelecky, V. A., Russell, N. 2011, Rev. Mod. Phys., 83, 11

Kowalski-Glikman, J., Nowak, S. 2002, PLB, 539, 126

Lewis, A., Bridle, S. 2002, PRD, 66, 103511

Linder, E. V. 2003, PRD, 68, 083504

Mattingly, D. 2005, Living Reviews in Relativity, 8, 5

Magueijo, J., Smolin, L. 2002, PRL, 88, 190403

Pan, Y., Cao, S., Gong, Y. G., Liao, K., Zhu, Z. H. 2013, PLB, 718,699

Pan, Y., Gong, Y. G., Cao, S., Gao, H., Zhu, Z. H. 2015, ApJ, 808,1

Passos, E., Anacleto, M. A., Brito, F. A., et al. 2016, PLB, 772, 870

Percival, B, A., et al. 2010, MNRAS, 401, 2148

Perlmutter, S., Aldering, G., Goldhaber, G., et al. 1999, ApJ, 517,565

Qi, J. Z., Zhang, M. J., Liu, W. B., 2016, arXiv:1606.00168

Qi, J. Z., Cao, S., Zhang, S. X., et al. 2019, MNRAS, 483, 1104

Ratra, B., Peebles, P. J. E. 1988, PRD, 37, 3406

Rodriguez M, M., Piran, T. 2006, JCAP, 0604, 006

Sarkar, S. 2002, MPLA, 17, 1025

Sefiedgar, A. S., Nozari, K., Sepangi, H. R. 2011, PLB, 696, 119

Seikel, M., Clarkson, C., \& Smith, M. 2012, JCAP, 1206, 036

Spergel, D. N., Verde, L., Peiris, H. V., et al. 2003, ApJS, 148, 175

Spergel, D. N., Bean, R., Doré, O., et al. 2007, ApJS, 170, 377

Tegmark, M., Blanton, M., Strauss, M., et al. 2004, ApJ, 606, 702

Vlasios, V., Jonathan, G., Tsvi, P., Amelino-Camelia, G., 2015,

Nature Physics, 11, 344

Vacaru, S. I. 2012, Gen. Rel. Grav, 44, 1015

Wei, J. J., Gao, H., Wu, X. F., Mészáros, P. 2015, PRL, 115, 261101

Zou, X. B., Deng, H. K., Yin, Z. Y., Wei, H. 2018, PLB, 776, 284

Kyle, F. K., Harriet, L. D., Heeyoung, O., et al. 2017, ApJ, 838, 2

Wei, J. J., Zhang, B. B., Shao, L., Wu, X. F., Mészáros, P. 2017, ApJL, 834, L13

Weinberg, D. H., Mortonson, M. J., Eisenstein, D. J., Riess, C. H. J., Rozo, E. 2013, PhR, 530, 87

Xu, D., Malesani, D., Fynbo, J. P. U., et al. 2016, GRB Coordinates Network, 19600

Yu, H., Wang, Y. F. 2016, ApJ, 828, 85

Yang, T., Guo, Z. K., Cai, R. G. 2015, PRD, 91, 123533

Zhang, M. J., Xia, J. Q. 2016, JCAP, 12, 005

Zhang, B. B., Zhang, B., Castro-Tirado, A. J., et al. 2016, arXiv:1612.03089

Zhang, S., Ma, B. Q. 2015, ApJ, 61, 108

Zhang, Y., Liu, X. W., Qi, J. Z., Zhang, H. S. 2018, JCAP, 08, 027

Zheng, X. G., Qi, J. Z., Cao, S., et al. 2019, EPJC, 79, 637

Zou, X., Deng, H., Yin, Z., Wei, H. 2018, PLB, 776, 284 